\title{
Modified Characteristics of Hadronic Interactions
}

\author{
J. Blažek, ${ }^{a, *}$ J. Vícha, ${ }^{a}$ J. Ebr, ${ }^{a}$ R. Ulrich, ${ }^{b}$ T. Pierog ${ }^{b}$ and P. Trávníček ${ }^{a}$ \\ ${ }^{a}$ Institute of Physics of the Czech Academy of Sciences (FZU), Prague, Czech Republic \\ ${ }^{b}$ Karlsruhe Institute of Technology (KIT), Institute for Astroparticle Physics, Karlsruhe, Germany \\ E-mail: blazekj@fzu.cz
}

The development of hadronic cascades in extensive air-showers is modeled by hadronic interaction models based on extrapolations of accelerator data. The models' predictions at the highest energies (above $\sim 10^{18} \mathrm{eV}$ ) are at a known tension with the description of measurements of the muonic component, if the mass composition derived from the fluorescence detection technique is assumed. We apply an ad-hoc modification to the CORSIKA Monte-Carlo generator that allows for adjustment of features of hadronic interactions such as multiplicity, elasticity and cross-section. Compared to similar previous studies, we are now able to obtain not only information related to the longitudinal development of the shower, such as the mean depth of shower maximum, but also information about the lateral distribution of particles. Moreover, we generate a scan across the various possible combined modifications of the Sibyll $2.3 \mathrm{~d}$ model using proton-initiated showers, quantify their effects on both the lateral and longitudinal features of a cosmic-ray shower and identify regions of the modification phase space which are promising for the explanation of both the ground-based and fluorescence-based measurements of cosmic rays at the highest energies.

$37^{\text {th }}$ International Cosmic Ray Conference (ICRC 2021)

July 12th - 23rd, 2021

Online - Berlin, Germany

\footnotetext{
${ }^{*}$ Presenter
} 


\section{Introduction}

The two largest experiments measuring the ultra-high energy cosmic rays (UHECR), the Pierre Auger Observatory [1] and the Telescope Array [2], have reported a tension between the mass composition extracted from the fluorescence measurement of the development of the shower in the atmosphere and the composition extracted from ground-based observation [3], [4]. In particular, the surface measurements point to a heavier composition of the primary beam due to an excess of muons when compared to Monte-Carlo simulations of primary particles consisting of species reported by the fluorescence technique through the observed depth of shower maximum $X_{\max }$. Such tension is corroborated by data reported by various other experiments measuring the UHECR [5].

The interpretation of such data always relies on the predictions of models of hadronic interactions which are themselves based on very precise measurements performed at accelerators, but then extrapolated to the energy and centrality region relevant to UHECR interactions. It follows that estimating the associated systematic uncertainty, e.g., in the mass-sensitive parameter $X_{\max }$, is difficult. For example, three commonly used hadronic interaction models, Sibyll 2.3d, QGSJET II-04 and EPOS-LHC, exhibit a spread of about $30 \mathrm{~g} / \mathrm{cm}^{2}$ consistently across the energy range $10^{18.0}-10^{19.0} \mathrm{eV}$. But this tells us little of what happens when we use a slight modification of a given model which would still be consistent with accelerator data but would result in an unknown effect on the features of an extensive air-shower. A natural question then arises: could any such modification consistently describe the muon tension described above? In this work, we will attempt to answer a simple case of a proton primary, the SIBYLL 2.3d interaction model and three ad-hoc modifications of hadronic interactions: the cross-section, elasticity, and the secondary multiplicity, and their combinations.

\section{Modifications of Hadronic Interactions}

Instead of altering the internal parameters of the hadronic interaction models, which was shown to explore only a small part of the modification phase space, we follow closely the scheme set-up by [6] - the major difference being the use of the CORSIKA Monte-Carlo generator [7], allowing access to 3-dimensional quantities such as the muon density far from the shower core, which was not possible in the 1-dimensional simulations of [6] using the CONEX package [8].

We make an ad-hoc modification to a regular output of the SiBYLL 2.3d hadronic generator, recalculating one of the following quantities:

- The hadronic particle cross-section, $\sigma_{\text {prod }}$.

- The elasticity, defined as $\kappa_{\mathrm{el}}=E_{\text {leading }} / E_{\text {tot }}$, where $E_{\text {leading }}$ is the energy of leading particle in the lab system and $E_{\text {tot }}$ is the energy of the incoming particle in the interaction.

- The secondary multiplicity, defined as the total number of particles escaping the interaction.

We also introduce an energy dependent energy factor in the same way as [6] in order to alter the hadronic interaction features in a scaling way:

$$
f\left(E, f_{19}\right)=1+\left(f_{19}-1\right) \cdot F(E)
$$


where $F(E)=0$ below some threshold energy $E_{\mathrm{thr}}$ and otherwise

$$
F(E)=\frac{\log _{10}\left(E / E_{\mathrm{thr}}\right)}{\log _{10}\left(10 \mathrm{EeV} / E_{\mathrm{thr}}\right)} .
$$

The functional form of the scaling is meant to represent the increasing uncertainty of the model extrapolation with energy, up to the factor $f_{19}$ at the reference energy $10 \mathrm{EeV}$. The technical implementation of the modifications is described in detail in [6] and we don't have the space to reproduce it here and will settle with a simple qualitative description. During the rescaling of the cross section $\sigma_{\text {prod }}$, the interaction length of the particles in the atmosphere is changed accordingly. This also affects the first interaction point of the primary particle of the shower. The rescaling of elasticity leads to a redistribution of the energy carried by the leading particle amongst the secondary particles of the interaction, whilst conserving the total energy. Finally, the rescaling of multiplicity leads to a change in the total number of produced particles. Particles are removed if $f_{19}<0$ and created if $f_{19}>0$. Energy conservation is enforced for every particle type separately, in order to avoid unphysical effects breaking quantum numbers which are otherwise conserved in strong interactions.

We now investigate how to choose the value of $E_{\mathrm{thr}}$. In [6], the value was set to $E_{\mathrm{thr}}=10^{15} \mathrm{eV}$, since that roughly corresponded to the most up-to-date Tevatron measurements at that time. The phase space was then chosen to be very wide, leading to extreme modifications with $f_{19}$ ranging from 0.2 to 5 . In this work, we aim to adopt a much more conservative approach. Ideally, we would like to set the threshold energy slightly below a known measurement at energy $E_{\text {ref }}$ and extrapolate the maximally allowed $f_{19}$ at $E=10^{19} \mathrm{eV}$ such that the modified quantity differs at most by three $\sigma$ at $E_{\text {ref }}$ from the measurement. This is most straightforwardly done for the proton-air (or pion-air) cross-section. The TOTEM collaboration has published a p-p inelastic cross-section at approximately $E_{\text {ref }}=10^{16.9} \mathrm{eV}$ in the lab frame with an uncertainty of roughly $2 \%$ [9]. At the same time, there is a measurement of the $\mathrm{p}-\mathrm{Pb}$ cross section from the CMS collaboration at roughly $E_{\text {ref }}=10^{16.1} \mathrm{eV}$ with $4 \%$ uncertainty [10]. The p-p and $\mathrm{p}-\mathrm{Pb}$ cross sections are in good agreement when compared using a simple Glauber model, strongly indicating the p-air cross section is also similarly constrained. Setting $E_{\mathrm{thr}}=10^{16} \mathrm{eV}$ and demanding a 3-sigma deviation from the TOTEM measurement, we arrive at the modification factor range of $f_{19} \subset(0.8,1.2)$, a much smaller interval than the one employed previously by [6].

For multiplicity there is more freedom since we do not have full coverage in the pseudorapidity region even in p-p collisions and there is no p-air multiplicity measurement at the relevant energies. For pseudorapidity up to 2 , where we have coverage [11], the predictions of the various hadronic interaction models differ by about $20 \%$. For pseudorapidity of 5, in a region not covered by data, the predictions differ by up to $50 \%$. Choosing a middle ground, we set the modification threshold such that $E_{\mathrm{thr}}=10^{15} \mathrm{eV}$ and the corresponding range of $f_{19} \subset(0.6,1.7)$. For elasticity, we have more freedom still, since there is no accelerator data in the relevant region - probably the most stringent limits actually come from cosmic ray experiments such as nuclear emulsion chambers [12]. We thus chose $E_{\mathrm{thr}}=10^{14} \mathrm{eV}$ and $f_{19} \subset(0.6,1.5)$. For lower values of $f_{19}$, the change in the modification effect is becoming negligible, for higher values we reach a saturation (mean elasticity in air-shower development is approximately equal to 0.4 and this factor becomes increasingly difficult to modify for factors of $f_{19} \sim 2$ ). 


\section{Simulations}

We simulate a proton primary particle with an energy of $7 \mathrm{EeV}$. This energy was motivated by precise hybrid measurements available at the Pierre Auger Observatory with sufficiently high statistics [13]. We simulate for five values of zenith angle distributed uniformly in $\cos ^{2}\left(0^{\circ}\right)-$ $\cos ^{2}\left(60^{\circ}\right)$. The CORSIKA Monte-Carlo generator [7] is compiled with the CONEX option and modified such that it can make use of the resampling code present in the standalone CONEX Monte-Carlo generator, with additional changes to allow for simultaneous modification of multiple parameters of the interactions and for a more fine-grained configuration of the process. We make use of the SibYLl 2.3d hadronic interaction model. We have performed tests simulating a small subset of the modification phase space also using the models QGSJET II-04 and EPOS-LHC and there appears to be a shift only in the overall normalization of the various observables, but the shape of the dependence on $f_{19}$ appears to be universal across models. This is to be expected, since the ad-hoc modifications are, by design, blind to the internal workings of a model.

The simulation is set-up as follows: the first several energy decades of the shower development are treated by the one dimensional CONEX full Monte-Carlo code, where each particle is tracked individually and every interaction is resampled if its energy is above the respective $E_{\mathrm{thr}}$. At $300 \mathrm{GeV}$, the hadronic particles are handed over to the CORSIKA code, which performs a 3D Monte-Carlo simulation and outputs the particle distributions at the observational level, which we set to roughly 1400 meters above the sea level to coincide approximately with the location of the Pierre Auger Observatory and Telescope Array. Muons produced at any point in the CONEX code are handed to the CORSIKA portion immediately. Crucially, in this way, we obtain also an information about the lateral distribution of the incoming particles and we extract the total number of muons and electromagnetic particles that are present at various distances from the shower core.

We sum the number of particles at ground in a thin ring (circular or elliptical, depending on the primary zenith angle) around a given distance from the shower axis, calculated in a plane perpendicular to this axis, regardless of their azimuth position - this quantity is roughly proportional to the signal detected by the various types of ground detectors. For example, this quantity read out at 1000 meters (let us denote it by $S^{*}(1000)$ ) would correspond to the quantity $S(1000)$ measured by the Pierre Auger Observatory. The exact factor of proportionality is of no interest for us, since we are interested only in the relative changes of the signal caused by the modifications to hadronic interactions, compared to a baseline, unmodified quantity. Since in the simulation we can perfectly separate the muonic and electromagnetic signal, and because we are mainly interested in studying the change of the muon content of the showers, we will work directly with $S_{\mu}^{*}(1000)$. We performed extensive tests in order to verify that the simulations obtained in this hybrid approach are compatible within statistical uncertainties with simulations performed with the full 3D simulation using the standalone CORSIKA treatment, in particular that the shapes of the lateral distribution functions exhibits a good match.

To summarize, we carry out simulations for five values of zenith angle, with three modifications of the cross-section $f_{19}^{\sigma}=(0.8,1.0,1.2)$, five modifications of the elasticity $f_{19}^{\text {el }}=$ $(0.6,0.8 ., 1.0,1.2,1.5)$ and five modifications in multiplicity $f_{19}^{\text {mult }}=(0.6,0.8,1.0,1.3,1.7)$, covering a total phase space of 375 combinations. We simulate 500 showers for every such bin. As our observables, we take the mean of the shift in $X_{\max }: \delta X_{\max }=X_{\max }-X_{\max }^{\text {ref }}$ and the mean of the 
muon rescaling: $R_{\mu}=\mathrm{S}_{\mu}^{*}(1000) / S_{\mu}^{*(\mathrm{ref})}(1000)$, where the reference values are determined from the unmodified simulations in which $f_{19}^{\sigma}=f_{19}^{\text {el }}=f_{19}^{\text {mult }}=1$.

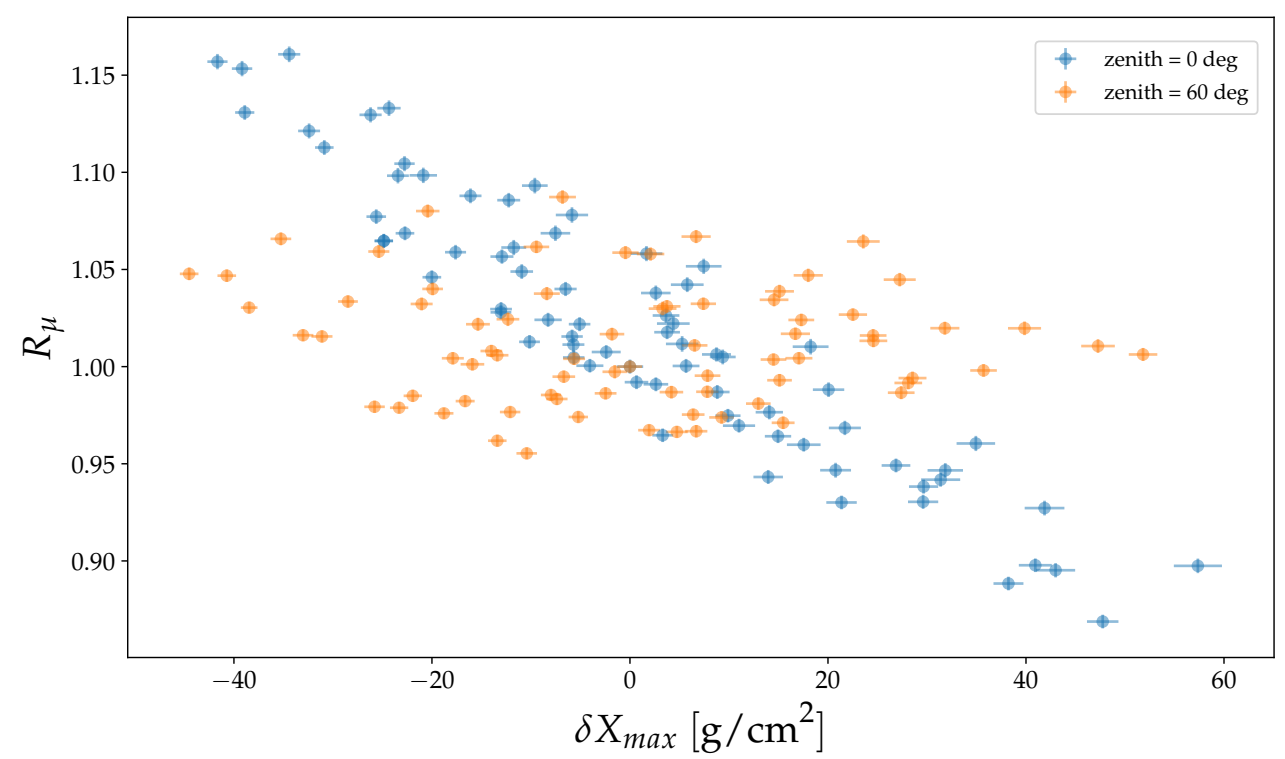

Figure 1: The effect of modifications of hadronic interactions in the $\delta X_{\max }-R_{\mu}$ plane shown for the two extreme zenith angles. Every point represents the mean values for 500 showers simulated with the unique combination of $\left(f_{19}^{\sigma}, f_{19}^{\text {el }}, f_{19}^{\text {mult }}\right)$ related to the baseline value $(1,1,1)$ for the given zenith angle.

\section{Results}

The first interesting question is that of the overall size of the phase space and the maximally allowed extent of the change of the observables $R_{\mu}$ and $\delta X_{\max }$. Figure 1 summarizes the results for the lowest and highest zenith angles. The three remaining zenith angles are not plotted in order to retain visual clarity, but the corresponding distributions lie between the two shown populations. It becomes immediately obvious that a change in $X_{\max }$ of similar magnitude is universally possible across all zenith angles. This is to be expected, since $X_{\max }$ is to a good approximation independent of zenith angle and the magnitude of the $X_{\max }$ shift depends mainly on the modifications of the cross-section, as we will show later. Where we see a strong dependence on zenith angle is however with the muon rescaling factor $R_{\mu}$.

Another interesting question is that of the effect of separate modifications $f_{19}^{\sigma}, f_{19}^{\text {el }}$ and $f_{19}^{\text {mult }}$. In [6] it was shown what happens to $X_{\max }$ and the total number of muons after modifications of the form such as, for example, $\left(f_{19}^{\sigma}, 1,1\right)$. Here we shall take a look at the entire combined phase space. In order to deal with the zenith dependence of $R_{\mu}$ and to retain as much information as possible, we average $R_{\mu}$ over all zenith angles for each unique combination of the phase space parameters. Thanks to the choice of the values to be uniformly distributed in $\cos ^{2}$, each value represents an angular bin of equal exposure for a horizontal detector. We plot the results in Figure 2. In every part of the figure, we color-code information about a different modification factor.

Reading the top Figure it becomes clear that a change in $f_{19}^{\sigma}$ translates directly to a shift in $X_{\max }$, as the whole distribution is shifted along the $\mathrm{x}$-axis. Increasing the cross-section, and subsequently 

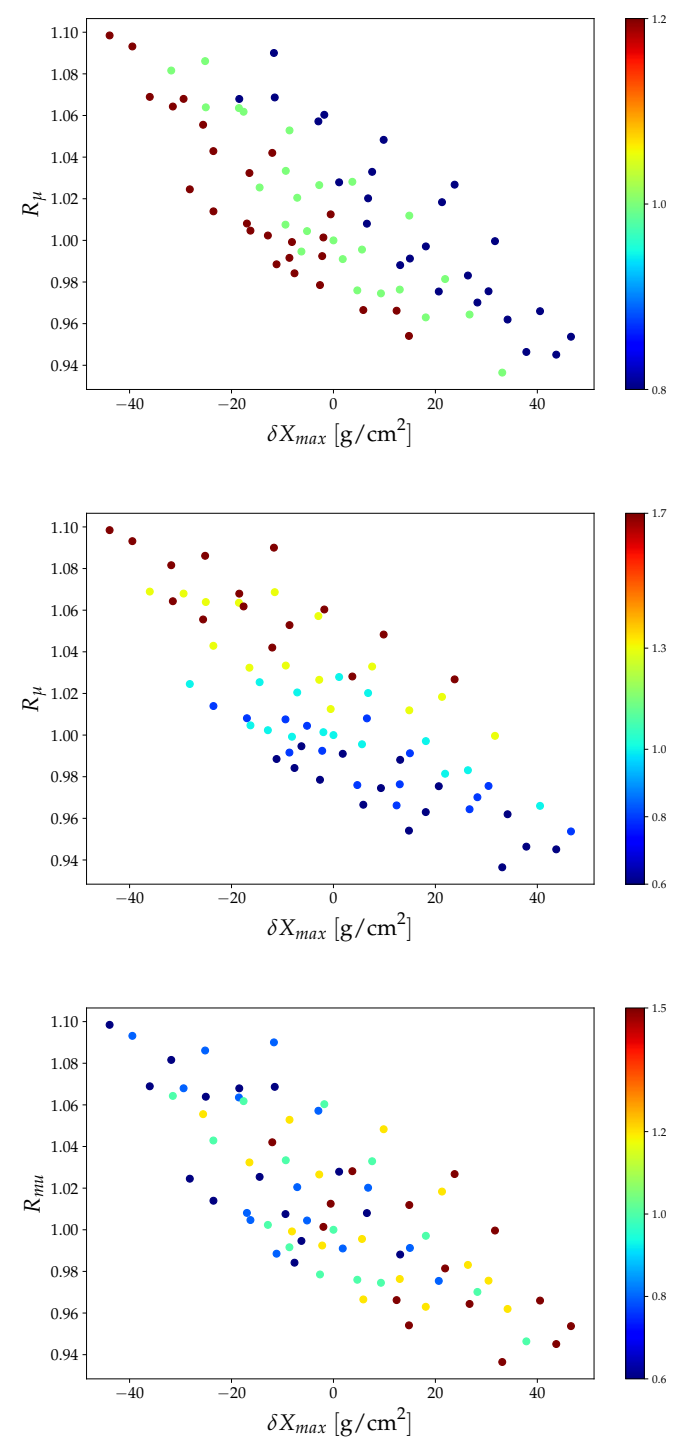

Figure 2: The effect of various types of modifications on $\delta X_{\max }$ and $R_{\mu}$. Every point is an average over all zenith angles. Color-coding is as follows: $f_{19}^{\sigma}$ (top), $f_{19}^{\text {mult }}$ (middle) and $f_{19}^{\text {el }}$ (bottom).

the interaction length, leads to shallower showers exactly as one would expect. A similar situation can be seen in the middle Figure. Here a change in multiplicity translates directly to the change in $R_{m u}$, as the whole distribution is shifted along the y-axis. Increasing the hadronic multiplicity leads to an increased number of muons on ground, as expected. The situation is less clear on the bottom Figure, where the interaction resampling and re-distribution of particle energy towards (or away) from the leading particle also effectively mimics a change in cross-section. Modifying the elasticity moves the whole distribution along the anti-diagonal.

Unlike in [6], which has dealt exclusively with modifications of single aspects of the interactions, we have explored a 3-dimensional space of parallel modifications of cross-section, elasticity and multiplicity. It is thus interesting to ask whether the results for the observables are a trivial 
combination of individual changes or not. To this end, we have, at a given zenith angle, for each set of simulations that differ only in one of the values of $f_{19}^{\sigma}, f_{19}^{\text {mult }}$ or $f_{19}^{\text {el }}$ (bottom) and fitted a straight line to the dependence of $\delta X_{\max }$ and $R_{\mu}$ on this variable. The dependence of the slope of these lines on the remaining two variables shows the magnitude of the interplay between the different modifications. For most choices, this interplay is modest - the strongest trend is exhibited when changing only $f_{19}^{\sigma}$, as shown in Figure 3, which clearly shows that the effect of the modification of the cross section depends on the magnitude of the modification of elasticity.
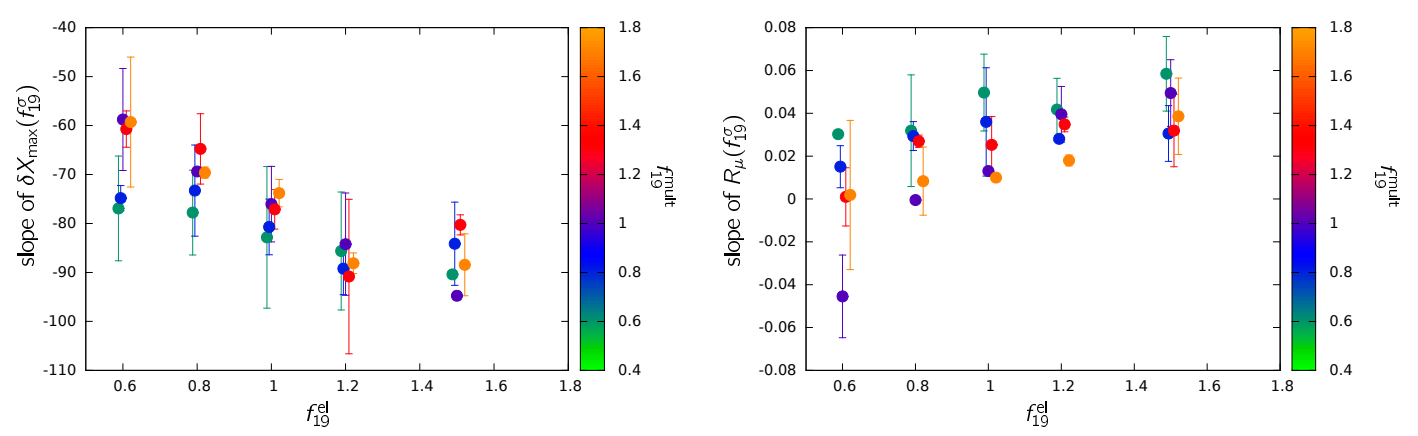

Figure 3: Slopes of lines fitted to the dependencies $\delta X_{\max }\left(f_{19}^{\sigma}\right)$ (left) and $R_{\mu}\left(f_{19}^{\sigma}\right)$ (right) for sets of simulations that differ only in the modification of cross-section $f_{19}^{\sigma}$ for zenith angle 0 degrees and their dependence on the modifications of multiplicity and elasticity $f_{19}^{\text {mult }}$ or $f_{19}^{\text {el }}$. Small offsets in the x-axis are added to the points for increased readability.

\section{Discussion}

We have shown that applying general modifications to hadronic interactions within the CORSIKA simulation package (using the CONEX option) affects not only the depth of the shower maximum and the overall number of muons produced (as already shown in the 1-dimensional simulations of [6]), but also the number of muons at ground at distances from the shower axis that are relevant for the detection at UHECR experiments (using the example of 1000 meters). While the numbers of muons predicted by simulations modified within reasonable limits taken from current accelerator measurements are generally not high enough to reproduce values seen previously in data by the Pierre Auger Observatory [3], the results are promising when compared to the recent work of the same collaboration [14].

The ability of parallel ad-hoc modification of different parameters of hadronic interactions in the full 3-D simulation package CORSIKA opens a multitude of possibilities for future work. We intend to carry out simulations for heavier primaries than proton and to use other hadronic interaction models besides SIBYLL as baseline. Ultimately, we can extract much more information from these simulations than just $\delta X_{\max }$ and $R_{\mu}$ and determine which combinations of baseline model and modifications provide the best description of the avilable data. This information can then be used as a guidance for the improvement of the hadronic interaction models and for the understanding of hadronic interactions at UHECR energies that are not directly accessible to study at artificial accelerators. 


\section{Acknowledgements}

This work is funded by the Czech Science Foundation under the project GACR21 - 02226M and by the MEYS CR under the project LTT18004.

\section{References}

[1] A. Aab et al. [Pierre Auger Collaboration], The Pierre Auger Cosmic Ray Observatory,

[2] M. Fukushima et al. [Telescope Array Collaboration], Telescope array project for extremely high energy cosmic rays, Prog. Theor. Phys. Suppl. 151 (2003), 206-210.

[3] A. Aab et al. [Pierre Auger Collaboration], Testing Hadronic Interactions at Ultrahigh Energies with Air Showers Measured by the Pierre Auger Observatory, Phys. Rev. Lett. 117192001.

[4] R. Abbasi et al. [Telescope Array Collaboration], Study of muons from ultrahigh energy cosmic ray air showers measured with the Telescope Array experiment Phys. Rev. 98, 022002.

[5] H. P. Dembinski et al. [EAS-MSU, IceCube, KASCADE-Grande, NEVOD-DECOR, Pierre Auger, SUGAR, Telescope Array and Yakutsk EAS Array], Report on Tests and Measurements of Hadronic Interaction Properties with Air Showers, EPJ Web Conf. 210 (2019), 02004.

[6] R. Ulrich et al., Hadronic Multiparticle Production at Ultra-High Energies and Extensive Air Showers, Phys. Rev. D,83 (2011) 054026.

[7] D. Heck et al. CORSIKA: A Monte Carlo code to simulate extensive air showers, FZKA-6019.

[8] T. Bergmann et al., One-dimensional Hybrid Approach to Extensive Air Shower Simulation, Astropart. Phys. 26 (2007), 420-432.

[9] G. Antchev et al. [TOTEM], First measurement of elastic, inelastic and total cross-section at $\sqrt{s}=13 \mathrm{TeV}$ by TOTEM and overview of cross-section data at LHC energies, Eur. Phys. J. C 79 (2019) no.2, 103.

[10] V. Khachatryan et al. [CMS], Measurement of the inelastic cross section in proton-lead collisions at $\sqrt{s_{N N}}=5.02 \mathrm{TeV}$, Phys. Lett. B 759 (2016), 641-662.

[11] S. Acharya et al. [ALICE], Pseudorapidity distributions of charged particles as a function of mid and forward rapidity multiplicities in pp collisions at $\sqrt{s}=5.02,7$ and $13 \mathrm{TeV}$ [arXiv:2009.09434 [nucl-ex]].

[12] Wilk, G. \& Włodarczyk, Z., Inelasticity in hadron-nucleus collisions from emulsion chamber studies, Phys. Rev. D 59 (1998) 014025.

[13] A. Aab et al. [Pierre Auger Collaboration], Evidence for a mixed mass composition at the 'ankle' in the cosmic-ray spectrum, Phys. Lett. B. 762 (2016) 288.

[14] J. Vicha for the Pierre Auger Collaboration, Proc. of 37th ICRC 2021, PoS(ICRC2021)310. 